\title{
The successful treatment of hypercapnic respiratory failure with oral modafinil
}

This article was published in the following Dove Press journal:

International Journal of COPD

30 April 2014

Number of times this article has been viewed

\author{
Helen Parnell' \\ Ginny Quirke' \\ Sally Farmer' \\ Sumbo Adeyemo ${ }^{2}$ \\ Veronica Varney' \\ 'Respiratory Department, \\ ${ }^{2}$ Pharmacy Department, St Helier \\ Hospital, Carshalton, Surrey, UK
}

Correspondence: Veronica Varney Respiratory Department, St Helier Hospital, Wrythe Lane, Carshalton, Surrey SM5 IAA, UK

Tel +44208296240I

Fax +442082963163

Email veronica.varney@esth.nhs.uk
Abstract: Hypercapnic respiratory failure is common in advanced chronic obstructive pulmonary disease and is usually treated by nasal ventilation. Not all patients requiring such ventilation can tolerate it, with anxiety and phobia influencing their reaction, along with treatment failure. We report the case histories of six patients with hypercapnic respiratory failure who were at risk of death due to refusal of nasal ventilation or its failure despite ongoing treatment. We report their improvement with oral modafinil $200 \mathrm{mg}$ tablets used as a respiratory stimulant, which led to discharge, improved arterial blood gases, and offset further admissions with hypercapnic respiratory failure. This drug is licensed for narcolepsy and is said to stimulate the respiratory system via the central nervous system. Its use in respiratory failure is an unlicensed indication, and there are no case reports or studies of such use in the literature. Its respiratory stimulant effects appear better than those with protriptyline, which was a drug previously used until its production was discontinued. Our findings suggest that a study of modafinil in hypercapnic respiratory failure would be warranted, especially for patients with treatment failure or intolerance to nasal ventilation. This may offer a way of shortening hospital stay, improving outcome and quality of life, and reducing death and readmissions.

Keywords: COPD, chronic obstructive pulmonary disease, nasal ventilation, acidosis, modafinil, hypercapnic respiratory failure

\section{Introduction}

Chronic obstructive pulmonary disease (COPD) is a rising epidemic which is responsible for one in six deaths currently and is predicted to rise to one in three deaths by 2020. ${ }^{1}$ The disease is high cost, with advanced COPD patients having repeat admissions with average stays of 8-11 days. ${ }^{2}$ These admissions are due to exacerbations, with the longest stays for those with hypercapnic respiratory failure (HRF). ${ }^{3} \mathrm{HRF}$ is common in moderately severe cases with forced expiratory volume in 1 second $\left(\mathrm{FEV}_{1}\right) \leq 40 \%$ of predicted. Upon infection, these patients develop acidosis with $\mathrm{CO}_{2}$ retention which can usually be reversed by nasal intermittent positive pressure ventilation (NIPPV), antibiotics, steroids, nebulized bronchodilators and other standard treatments for COPD. ${ }^{4}$ At our hospital, NIPPV is commenced in HRF when $\mathrm{pH}<7.35$ and $\mathrm{PaCO}_{2}>8 \mathrm{kPa}$ or earlier if there is a change in conscious level. According to our local guidelines, inspiratory positive airway pressure (IPAP) and expiratory positive airway pressure (EPAP) are increased according to responses of the patients (initial pressures IPAP 8-10, EPAP 5-6). The carbon dioxide levels will often correct after an acute exacerbation, without the need for long-term NIPPV. For the individual patient at this stage, however, their disease progression and further damage from the exacerbation (with or without 
smoking) will lead them to more frequent admissions and episodes of HRF in the future. ${ }^{5}$ Each admission carries the risk of death, which before the advent of NIPPV and the use of central nervous system stimulants such as doxapram, carried a very high mortality in the first 48 hours. ${ }^{6}$ Since NIPPV, the mortality is much lower acutely and lives are saved, but readmissions of the same patients can become more and more frequent, with high hospital bed use. ${ }^{7,8}$

The use of long-term NIPPV is expensive in the UK. Acute hospital costs of ventilation per patient episode are $£ 13,163$. While home therapy costs $£ 4,909$ per annum, with a ventilator life span of only 5 years. ${ }^{9}$

Many patients, with time, become poorly compliant due to the effects of NIPPV on sleep quality, nasal blockage, and dryness of the airway, with sinus-like headaches. ${ }^{10}$ As a result, many minimize their use and remove the NIPPV after an average of 4 hours.

In the past, protriptyline was an oral respiratory stimulant that had use in respiratory failure either alone or in combination with NIPPV or cuirass, for example. ${ }^{11}$ This drug was suddenly discontinued in 2000 in the UK.

Modafinil is a successful drug licensed for narcolepsy and acts through the dopaminergic system and also has effects on the sympathetic nervous system. ${ }^{12-14}$ It has multiple sites of action on the central nervous system and is noted to have an effect on respiratory drive. ${ }^{15}$ This effect is probably via the brain stem respiratory center and mediated by the tuberomammillary nuclei, which activate the cerebral cortex, including the striatum of motor activity. ${ }^{16-18}$ Studies have shown actions in obstructive sleep apnea and apnea-hypopnea syndromes, and even shift workers. ${ }^{19}$ The license for shift work has not been renewed in the UK by Cephalon UK Ltd since 2010. The described actions of modafinil at various sites in the central nervous system led us to trial this drug in patients with HRF.

Modafinil must be avoided in pregnancy, lactation, and uncontrolled moderate to severe hypertension with cardiac arrhythmia. Patients with major anxiety need cautious use. There is evidence for low-level dependence on modafinil. ${ }^{20}$ Modafinil stimulates the brain to increase alertness and also to stimulate the rate of breathing. This latter effect has not been formally studied, although obstructive sleep apnea conditions have been examined; ${ }^{19,21}$ most studies focus on narcolepsy. ${ }^{22-24}$

We report the use of modafinil in our six most recent cases with HRF, where prolonged or repeat admissions were occurring or NIPPV treatment was refused, failed, or was not tolerated. Modafinil corrected the problem with very significant life quality improvements noted by the patients and their family, which avoided readmissions, as outlined in the case histories. The authors confirm that there is no conflict of interest in the presentation of these cases.

\section{Case I}

An 87-year-old man (Table 1) was admitted unconscious with HRF. This was his third admission for the same condition in 4 weeks. On each occasion, his blood gases were similar (Table 2). He was commenced on NIPPV and generally recovered consciousness later the same day. On awakening, he would panic and pull off the NIPPV mask and refuse to go on with the treatment. He would be observed for approximately 4 days and would remain hypercapnic (partial pressure of arterial carbon dioxide $\left[\mathrm{PaCO}_{2}\right]>6.8 \mathrm{kPa}$ ). Once judged clinically stable with optimized bronchodilatation, he would be discharged, only to return 1 week later unconscious in the same condition. On questioning, he denied any additional use of alcohol, sleeping tablets, tranquilizers, or painkillers prior to these episodes. He did not have home oxygen, and these episodes could not be explained by an infectious process. He had a home nebulizer (air driven) for salbutamol and ipratropium, a seretide 250 inhaler, frusemide, aspirin, digoxin, and oral theophylline. He was on mirtazapine $45 \mathrm{mg}$ once daily (od) for his anxiety, and citalopram $40 \mathrm{mg}$ od for several years without prior respiratory depression. He admitted to anxiety and panic disorders, even associated with using a nebulizer mask, and especially with the NIPPV mask.

He felt that his respiratory condition had generally deteriorated over 6 months, following pneumonia the previous summer. The onset of each episode of respiratory failure was unpredictable, and he would report going to bed well but awake in hospital on the NIPPV without any warning. He would not consider NIPPV even though the risks were explained. Low-flow nasal oxygen $(1 \mathrm{~L} / \mathrm{min})$ rapidly increased the $\mathrm{PaCO}_{2}$ to $>8.3 \mathrm{kPa}$. To avoid a further admission and the risk of death, he was started on modafinil $200 \mathrm{mg}$ in the morning. By 10 days of treatment, his arterial gases had improved, along with day- and nighttime oximetry (Table 2), and remained stable long-term. He had one admission with a myocardial infarction, without any respiratory concerns. Modafinil did not affect his previous anxiety, and he felt his breathing and well-being had improved. Lung function remained the same. In April 2013, he developed community-acquired pneumonia and died following admission, without HRF after 15 months of treatment.

\section{Case 2}

An 81-year-old man (Table 1) was admitted with a left hip fracture after his leg suddenly gave way at home. He had 
Table I Demographics of the six patients including spirometry and past medical history

\begin{tabular}{|c|c|c|c|c|c|c|}
\hline & Case I & Case 2 & Case 3 & Case 4 & Case 5 & Case 6 \\
\hline 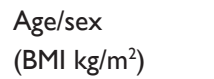 & $87 / M(23)$ & $8 \mathrm{I} / \mathrm{M}(23)$ & 68/M (30) & 64/M (38) & $78 / F(18)$ & $66 / F(52)$ \\
\hline $\begin{array}{l}\text { Diagnosis } \\
\text { (duration, years) }\end{array}$ & COPD (15) & COPD (I2) & COPD (1.5) & COPD (6) & COPD (10) & $\mathrm{PTH}^{*}(4)$ \\
\hline $\begin{array}{l}\text { Smoking status } \\
\text { (pack-years) }\end{array}$ & 4/d (70) & $\begin{array}{l}\text { Ex-smoker } \\
15 \text { years }(50)\end{array}$ & $30 / d(60)$ & $30 / d(60)$ & $\begin{array}{l}\text { Ex-smoker } \\
3 \text { years }(50)\end{array}$ & Passive \\
\hline $\begin{array}{l}\text { FEV } \\
\text { (\% predicted) }\end{array}$ & $0.95(41 \%)$ & $0.69(26 \%)$ & $0.95(37 \%)$ & 1.12 (33\%) & $0.42(24 \%)$ & $0.73(43 \%)$ \\
\hline $\begin{array}{l}\text { FVC } \\
\text { (\% predicted) }\end{array}$ & I.78 (55\%) & $1.84(51 \%)$ & 2.14 (54\%) & $2.76(64 \%)$ & I.05 (45\%) & I.I (45\%) \\
\hline $\begin{array}{l}\text { PEFR } \\
\text { (\% predicted) }\end{array}$ & $253(54 \%)$ & 161 (36\%) & I 88 (44\%) & $205(40 \%)$ & $100(2 \mid \%)$ & $80(26 \%)$ \\
\hline $\mathrm{PMH}$ & $\begin{array}{l}\text { AF } \\
\text { HTN } \\
\text { COPD }\end{array}$ & $\begin{array}{l}\text { COPD } \\
\text { OSA }\end{array}$ & $\begin{array}{l}\text { COPD } \\
\text { Aspirin allergy } \\
\text { Polycythemia }\end{array}$ & $\begin{array}{l}\text { COPD } \\
\text { Polycythemia }\end{array}$ & $\begin{array}{l}\text { TB } \\
\text { Plombage } 1950 \\
\text { Ca breast } \\
\text { HTN } \\
\text { Schizophrenia } \\
\text { Anxiety } \\
\text { Aortic stenosis }\end{array}$ & $\begin{array}{l}\text { Gross obesity } \\
\text { CCF } \\
\text { PTH } \\
\text { COPD }\end{array}$ \\
\hline Chest X-ray & COPD & COPD & COPD & COPD & $\begin{array}{l}\text { COPD, LUL } \\
\text { plombage, pleural } \\
\text { calcification }\end{array}$ & $\begin{array}{l}\text { Cardiomegaly and } \\
\text { dilated pulmonary } \\
\text { arteries* }\end{array}$ \\
\hline
\end{tabular}

Note: *Pulmonary hypertension.

Abbreviations: AF, atrial fibrillation; BMI, body mass index; Ca breast, breast cancer; CCF, congestive cardiac failure; COPD, chronic obstructive pulmonary disease; d, day; F, female; FEV , forced expiratory volume in I second; FVC, forced vital capacity; HTN, hypertension; LUL, left upper lobe; M, male; OSA, obstructive sleep apnea; PEFR, peak expiratory flow rate; PMH, past medical history; PTH, pulmonary hypertension; TB, tuberculosis.

COPD and had used a nasal CPAP (continuous positive airway pressure) machine (6 $\mathrm{cm}$ pressure without oxygen) for obstructive sleep apnea for 7 years. Medication included seretide (250), two puffs twice daily (bid) by a spacer, tiotropium $18 \mu \mathrm{g}$ mane, salbutamol MDI (metered-dose inhaler)
$200 \mu \mathrm{g}$ as required, carbocisteine $375 \mathrm{mg}$ three times a day, and frusemide $40 \mathrm{mg}$ od. Upon admission with the hip fracture, his oxygen saturations $\left(\mathrm{SpO}_{2}\right)$ were low (Table 2), with HRF. As a result, surgery was delayed, and nasal ventilation was commenced (IPAP 12, EPAP 6 ) with oxygen ( $2 \mathrm{~L} / \mathrm{min})$.

Table 2 Measures of hypercapnic respiratory failure and response to modafinil after 10 days

\begin{tabular}{|c|c|c|c|c|c|c|}
\hline & Case I & Case 2 & Case 3 & Case 4 & Case 5 & Case 6 \\
\hline \multicolumn{7}{|l|}{ Admission arterial gases (on air)* } \\
\hline $\mathrm{pH}$ & 7.32 & 7.33 & 7.33 & 7.45 & 7.22 & 7.35 \\
\hline $\mathrm{PaO}_{2}$ & 6.88 & 8.61 & 5.7 & 5.8 & 5.46 & 5.4 \\
\hline $\mathrm{PaCO}_{2}$ & 8.24 & 8.08 & 8.84 & 6.76 & 14.2 & 8.6 \\
\hline Daytime mean (air) saturations & $80 \%$ & $75 \%$ & $80 \%$ & $80 \%$ & $67 \%$ & $89 \%$ \\
\hline Nocturnal mean (air) saturations & No data & $65 \%$ & $77 \%$ & $66 \%$ & $72 \%$ & $75 \%$ \\
\hline NIPPV settings IPAP (EPAP) & $10(6)$ & $20(8)$ & Rejected & Rejected & $12(6)$ & $16(8)$ \\
\hline \multicolumn{7}{|l|}{ After 10 days of modafinil } \\
\hline Daytime mean (air) saturations & $91 \%$ & $94 \%$ & $92 \%$ & $92 \%$ & $93 \%$ & $90 \%$ \\
\hline Nocturnal mean (air) saturations & $87 \%$ & $90 \%$ & $85 \%$ & $76 \%$ & $87 \%$ & $81 \%$ \\
\hline \multicolumn{7}{|l|}{ Gases on air* after 10 days } \\
\hline $\mathrm{pH}$ & 7.44 & 7.61 & 7.42 & 7.41 & 7.48 & 7.45 \\
\hline $\mathrm{PaO}_{2}$ & 9.7 & 8.5 & 8.5 & 8.6 & 9.1 & 7.5 \\
\hline $\mathrm{PaCO}_{2}$ & 5.86 & 6.1 & 6.41 & 6.21 & 6.59 & 6.9 \\
\hline $\mathrm{AHI}$ & 28 & 2.6 & 69 & 31 & 8 & 83 \\
\hline $\begin{array}{l}\text { Duration without further HRF, } \\
\text { months }\end{array}$ & 15 & 15 & 21 & 36 & 10 & 17 \\
\hline Readmissions in HRF & No & No & No & $\begin{array}{l}\text { Yes with } \\
\text { exacerbation } \\
\text { after } 36 \text { months }\end{array}$ & $\begin{array}{l}\text { Yes at } \\
\text { death after } \\
60 \% \text { oxygen }\end{array}$ & No \\
\hline Cause of death & Pneumonia & Alive & Alive & Alive & Pneumonia & Alive \\
\hline
\end{tabular}

\footnotetext{
Note: *kPa.
}

Abbreviations: AHI, apnea-hypopnea index; EPAP, expiratory positive airway pressure; HRF, hypercapnic respiratory failure; IPAP, inspiratory positive airway pressure; NIPPV, nasal intermittent positive pressure ventilation; $\mathrm{PaO}_{2}$, partial pressure of arterial oxygen; $\mathrm{PaCO}_{2}$, partial pressure of arterial carbon dioxide. 
At 48 hours, gases improved, and he proceeded to have his hip replacement.

Postoperatively, the patient was observed in the high dependency unit, where nasal ventilation continued before transfer to the orthopedic ward for mobilization. By day 3 postop, he was still in HRF despite NIPPV (IPAP 18, EPAP 8) and oxygen $1 \mathrm{~L} / \mathrm{min}$.

The patient's chest was clear, without evidence of infection clinically or radiologically, but antibiotics and steroids were started in case of benefit. His arterial carbon dioxide levels remained elevated without painkillers, sedatives, oxygen over-prescription, or any obvious explanation for NIPPV failure.

He transferred to the chest ward and continued mobilizing with the physiotherapist, who noticed low $\mathrm{SpO}_{2}(68 \%)$ on exertion. Eventually, NIPPV use became continuous due to increasing episodes of $\mathrm{CO}_{2}$-narcosis by day, occurring when the ventilator mask was removed for breakfast, lunch, or supper. He would suddenly be noted by the nurses to be sleepy and unresponsive (respiratory rate 6-8 bpm), with $\mathrm{PaCO}_{2}$ values $>10 \mathrm{kPa}$ on air. All his pharmacological treatments and bronchodilators had been optimized. He had been rescued so many times from unconsciousness that there was great concern as to whether we could break this cycle. His family were alarmed and felt he could not be discharged in view of these episodes. NIPPV continued for over 6 weeks without clear benefit, despite compliance with treatment and increased inspiratory and expiratory pressure adjustments. A request to pharmacy for modafinil $200 \mathrm{mg}$ mane was granted. From the first tablet this completely stopped the episodes of unconsciousness and facilitated his discharge from hospital. By day 10, gases had improved (Table 2). He was carefully observed by frequent home visits by our respiratory nurses and has remained very well, without NIPPV or CPAP at any time. His oximetry and arterial gases improved (Table 2). On modafinil, there have been no further readmissions, no episodes of loss of consciousness, and no adverse effects in 15 months. The patient claims that he is better by the week and extremely active compared with previously. He is pursuing his hobbies of cooking and gardening, and he partakes in social outings.

\section{Case 3}

Case 3 is a 68-year-old man with HRF, diagnosed following admission for leg swelling. His hemoglobin was $20.3 \mathrm{~g} \mathrm{~m} / \mathrm{dL}$ (hematocrit 0.66) and he admitted to shortness of breath and a cough, with an exercise tolerance of 50 meters. The polycythemia was deemed secondary to smoking
(Table 1), and he admitted to lethargy and headache and had a cyanotic appearance. He was commenced on seretide (250) two puffs bid via a spacer, tiotropium $18 \mu \mathrm{g}$ mane, and salbutamol as required. Arterial gases confirmed HRF (Table 2). His findings were consistent with cor pulmonale and COPD. Overnight oximetry showed mean $\mathrm{SpO}_{2}$ of $77 \%$ on air, but he rejected NIPPV. Once clinically improved by bronchodilators, steroids, and diuretics, his arterial gases still showed HRF with significant hypoxemia. On $1 \mathrm{~L} / \mathrm{min}$ oxygen over 4 hours, his $\mathrm{PaCO}_{2}$ increased to $>8.84 \mathrm{kPa}$. His life quality had been poor, and he smoked to increase his state of alertness. He was commenced on modafinil $200 \mathrm{mg}$ in the morning, and by 14 days, he felt better, more alert, and stopped smoking. Oximetry improved (Table 2) along with his arterial gases. His forced vital capacity (FVC) improved on treatment and smoking cessation to $63 \%$ predicted. He remained awake by day and slept well at night. He no longer appears cyanosed, has had no further admissions in 21 months, and went on to complete pulmonary rehabilitation. He remains active and stable on modafinil $200 \mathrm{mg}$ mane, without adverse effects.

\section{Case 4}

A 64-year-old man (Table 1) was referred by the hematologists in 2009 for polycythemia considered secondary to his respiratory disease. They recorded low $\mathrm{SpO}_{2}$ and a hematocrit of 0.66 requiring venesection every 3-4 months. He was generally cyanosed, and an echocardiogram confirmed a dilated right heart consistent with cor pulmonale. A computed tomography scan had ruled out pulmonary emboli but showed hyperinflation without significant emphysematous changes. The patient was inactive, overweight, and smoking, with daily malaise and headaches. He took regular Symbicort ${ }^{\circledR}$ (AstraZeneca, London, UK) (400/12) two puffs bid, tiotropium 18 $\mu \mathrm{g}$ mane, and bumetanide.

Oxygen studies showed HRF (Table 2) and low nocturnal $\mathrm{SpO}_{2}$. An oxygen trial (on $1 \mathrm{~L} / \mathrm{min}$ nasal prongs) showed an improvement in oxygen levels to $77 \%$, with a small rise in $\mathrm{PaCO}_{2}$ at 2 hours to $6.91 \mathrm{kPa}$; but the overnight trial produced severe headache, increased drowsiness, and a further rise in $\mathrm{PaCO}_{2}$. In the UK, his active smoking would preclude any use of home oxygen even with a nasal ventilator. He would not contemplate NIPPV to improve both his oxygen and carbon dioxide levels. All the recommended smoking cessation advice had been given to no avail.

Modafinil $200 \mathrm{mg}$ mane was commenced in view of the difficulties. Day- and nighttime $\mathrm{SpO}_{2}$ improved (Table 2), with complete alleviation of malaise, headache, 
and inactivity. His cyanosis resolved and he looked pink. His $\mathrm{FEV}_{1}$ improved marginally (from $33 \%$ to $38 \%$ ), and also his FVC (from $61 \%$ to $85 \%$ ), but smoking continued. His wife reported that he developed an interest in things, and was much more alert and chatty for the first time in several years. The increased activity led to a 3 stone weight loss over 4 months. He continued to smoke but had no admissions in 3 years remaining on modafinil without requiring further venesections.

In December 2012, the patient developed a chest infection with leg edema. This did not improve over several weeks despite antibiotics, steroids, and diuretics, and there was an increase in hypoxia and drowsiness leading to admission. Gases showed $\mathrm{pH}$ 7.37, $\mathrm{PaCO}_{2} 7.94, \mathrm{PaO}_{2}$ (partial pressure of arterial oxygen) $5.6, \mathrm{Be}$ (base excess) $9, \mathrm{HCO}_{3}$ (bicarbonate level, $\mathrm{mmol} / \mathrm{L}$ ) 34.7, and $\mathrm{SpO}_{2} 63 \%$. He was treated with antibiotics, steroids, bronchodilators, mucolytics, physiotherapy, short-term NIPPV, and diuretics for right heart failure to which he responded. Since discharge, he has given up smoking and continued with his weight loss. He has remained on modafinil now for 4 years, with arterial gases remaining stable, apart from a single admission at 36 months. Nocturnal $\mathrm{SpO}_{2}$ is now $90 \%$ on air, with only a mild obstructive sleep apnea pattern. Daytime saturations remain at $92 \%$ on air. With smoking cessation and further weight loss, his spirometry improved further. $\mathrm{FEV}_{1}$ was $1.23 \mathrm{~L}$ (42\% predicted) and FVC $2.28 \mathrm{~L}$ (88\% predicted).

\section{Case 5}

A 78-year-old woman (Table 1) was admitted with HRF, with a presumed infective exacerbation. She also suffered memory loss and an anxiety disorder with schizophrenia. She had been on long-term antipsychotic therapy and was taking the following drugs: frusemide $40 \mathrm{mg}$ /day, losartan $25 \mathrm{mg}$ /day, olanzapine $10 \mathrm{mg}$ /day, mirtazapine $30 \mathrm{mg}$ /day, seretide (250) two puffs bid, and tiotropium bromide $18 \mu \mathrm{g} /$ day.

The patient's lung function was poor (Table 1), and following treatment, she was readmitted later the same month with a second exacerbation of HRF (C-reactive protein 68). Her arterial gases were similar on both admissions (Table 2). Although she responded to NIPPV each time, she was intolerant due to claustrophobia associated with wearing the mask, removing it as soon as she was alert. Once she was alert and more stable, overnight oximetry showed mean nocturnal $\mathrm{SpO}_{2}$ of $72 \%$ on air. Oxygen studies even at low flow $1 \mathrm{~L} / \mathrm{min}$ (nasal cannulae for 2 hours) gave a rise in $\mathrm{PaCO}_{2}$ to $11.0 \mathrm{kPa}$. To facilitate discharge and prevent a third admission, modafinil $200 \mathrm{mg}$ mane was commenced with monitoring in view of her blood pressure, anxiety, and psychosis. At 10 days following discharge on modafinil her arterial gases had improved (Table 2). The prescription was continued, and the patient remained entirely well and out of hospital. There were no adverse events of modafinil on her anxiety or her mental health, and ongoing follow-up continued for 10 months. The improvement was maintained despite no discernable change in her lung function.

In December 2012, the patient developed communityacquired pneumonia with congestive cardiac failure. On hospital transfer, the ambulance crew gave $60 \%$ oxygen, resulting in a rise in the $\mathrm{PaCO}_{2}$ to $10.1 \mathrm{kPa}$. This was corrected with NIPPV, and she was treated for heart failure and pneumonia but died within 24 hours.

\section{Case 6}

A 66-year-old retired National Health Service (NHS) nursing sister (Table 1) was admitted July 2012 unconscious with HRF. Arterial gases on admission are shown in Table 2. She was a nonsmoker, but as a nurse had been exposed to years of passive smoking at work and at home. Her spirometry was poor, with a restrictive pattern, probably due to her increased body mass index. Clinically, she was considered to have COPD, congestive cardiac failure, and pulmonary hypertension. In the past, she had been under the cardiologist with a diagnosis of congestive cardiac failure. Treatment included frusemide $40 \mathrm{mg}$ od, Symbicort (400/12) two puffs bid, tiotropium $18 \mu \mathrm{g} /$ day, and candesartan $4 \mathrm{mg} /$ day.

The patient described being unwell and housebound during the last 4 years, ordering shopping online, with lethargy and shortness of breath probably due to chronic elevation in her blood carbon dioxide. She suffered chronic leg edema and was aware that her heart was large on chest X-ray, with dilated pulmonary arteries. Her echocardiogram showed a normal left ventricle but a dilated right ventricle, with tricuspid regurgitation but preserved right ventricle function. The estimated pulmonary pressure was $81 \mathrm{mmHg}$. The patient was obese and had been an alcoholic but had given up drinking alcohol 30 years before. She was commenced on continuous NIPPV, and after several days, she improved clinically. She then developed severe nose bleeds each night while continuing nocturnal NIPPV, despite cauterization, oral tranexamic acid, and stopping prophylactic heparin. The bleeding ceased once NIPPV was stopped.

Day and night oximetry recordings improved after 1 week of NIPPV (Table 2 ) from $75 \%$ on air initially. Dietary advice and exercise was highlighted. The idea of lifelong NIPPV did 
not appeal to her despite the explained benefits to symptoms and survival, along with the improvement in her arterial gases and energy.

Overnight studies on $1 \mathrm{~L} / \mathrm{min}$ of oxygen via nasal prongs showed her to be very oxygen sensitive, with a sharp rise in her $\mathrm{PaCO}_{2}$ to $>9 \mathrm{kPa}$. Due to the risks of further HRF, she was commenced on oral modafinil $200 \mathrm{mg}$ mane. At 10 days following discharge from hospital, arterial blood gases had improved (Table 2).

The patient felt well, her fatigue and headaches cleared, and she described herself as being "champion" compared with the last 4 years. Her progress continued, and she was out of the house doing her own shopping, which aided weight loss. She has had no further admissions in HRF and remains on modafinil (17 months).

Over the last severe winter, she became very inactive, gaining more weight (13 kg), leading to a fall in spirometry. $\mathrm{FEV}_{1}$ was $0.6 \mathrm{~L}(36 \%)$ and FVC was $0.64 \mathrm{~L} \mathrm{(31 \% ),} \mathrm{but} \mathrm{arte-}$ rial gases remained stable. As a result, she is undergoing pulmonary rehabilitation and further dietary advice. She did have a referral for nasal ventilation which she had repeatedly deferred.

\section{Discussion}

Searches of the current medical literature show that there are no described studies or case reports of modafinil use in HRF. Our findings show modafinil to have had a beneficial effect on all the described patients, improving their oxygenation and lowering their blood carbon dioxide. This effect occurred without a clear change in lung function, and the likely mechanism is unclear. The patients remained stable and without readmissions with HRF for periods of over 1 year, and we encountered no adverse events. The possible effects on respiratory rate have not been formerly studied, with only one patient (case 2) showing an alkalosis on their arterial gases post-modafinil. In case 2, around-theclock treatment with NIPPV at maximal ventilation over 6 weeks did not correct the blood carbon dioxide level. Yet modafinil improved his arterial gases measured by 10 days of treatment, which is the time required for a steady-state blood level. From our cases, the effects of modafinil on HRF would appear useful and needs evaluation, especially with the increase in pressure to reduce admissions and length of stay, especially in advanced COPD. It may be useful in HRF unrelated to COPD, as in case 6, especially where the etiology of the HRF was probably mixed.

Modafinil $200 \mathrm{mg} /$ day has a cost of $£ 745.92$ per annum within the NHS (actual cost per annum is $£ 1,262$ ). This is relatively little compared with the average cost of admission for 10 days. The current NHS tariff for patients admitted with COPD who require NIPPV is $£ 2,227-£ 3,228$ per episode, depending on complications. These tariffs are usually a recurring feature in this disease but probably insufficient. The real cost of NIPPV per inpatient episode in the UK, from the Leeds study 10 years ago, gave a value of $£ 13,163 .{ }^{9}$ The cost savings are obvious from the cases we described, and has probably saved lives and hospital bed occupancy in the presence of the high admission rates for advanced COPD.

Additionally, there are calls for current drugs on the market to have their full therapeutic potential investigated instead of a relentless drive to manufacture new agents. ${ }^{25-28}$ This has been highlighted by many serendipitous observations of drug benefit in unrelated conditions, leading to major therapeutic breakthroughs in disease management. One such example is the use of thalidomide in myeloma. ${ }^{29}$

However, the restrictive practices placed on doctors by evidence-based medicine makes off-license use difficult. In the UK, restricted drug use curtails completely new treatment innovations for patient benefit. This includes preliminary observations and testing, which is always advisable prior to time and resources being spent on clinical trials.

In the USA, the "off-label" use of modafinil is very common by doctors and is acknowledged in the medical journals, with a 15-fold increase in off-license use by neurologists, pulmonologists, otolaryngologists, and psychiatrists..$^{30,31}$ Currently, the only evidence-based treatment in HRF is the use of oral acetazolamide which produces a decrease in blood carbon dioxide levels, maybe via actions in the carotid body and elsewhere. ${ }^{32}$

To take this work forward, we feel that an open study of modafinil in HRF is justified, probably using modafinil added to standard care versus standard care alone. This could include non-COPD cases of HRF. Many patients, including those with COPD, have abnormalities in sleep architecture and oximetry, which is being increasingly recognized, indicating a more complex picture in COPD. ${ }^{33}$ The speed of patient improvement in arterial gases, overnight oximetry, and hospital length of stay/readmission would no doubt be of interest to future clinical commissioning groups in the National Health Service. Other interesting questions would include the drugs effect on the respiratory rate, quality of life, sleep architecture, and BODE (body mass index, airflow obstruction, dyspnea, and exercise capacity) score. This would require a formal study in order to take a more systematic approach to data collection. 


\section{Disclosure}

The authors report no conflicts of interest in this work.

\section{References}

1. Dept of Health Care. Facts about COPD. UK Government Web Archives. 2010;11943:1-2.

2. Utens CM, Maarse JA, van Schayck OC, Maesen BL, Rutten MP, Smeenk FW. Care delivery pathways for chronic obstructive pulmonary disease in England and the Netherlands: a comparative study. Int $J$ Integrated Care. 2012;12:1-9.

3. Schmaker GL, Epstein SK. Managing acute respiratory failure during exacerbation of chronic obstructive pulmonary disease. Resp Care. 2004;49(7):766-776.

4. Gruffydd-Jones K, Loveridge C. The 2010 NICE Guidelines: how do they compare with the GOLD Guidelines. Prim Care Respir J. 2011;20(2):199-204.

5. NHS London; COPD Factsheet: - Strategic needs assessment 2010-2011. NHS website. 2011:1-12.

6. Moser PS, Luchsinger PC, Adamson JS, et al. Respiratory stimulation with intravenous doxapram in respiratory failure - a double-blind cooperative study. $N$ Engl J Med. 1973;288:427-431.

7. Marchetti N, Criner GJ, Albert RK. Preventing acute exacerbations and hospital admissions in COPD. Chest. 2013;143(5):1444-1454.

8. Doherty MJ, Greenstone MA. Survey of non-invasive ventilation (NIPPV) in patients with acute exacerbations of chronic obstructive pulmonary disease (COPD) in the UK. Thorax. 1998;53:863-866.

9. Tuggey JM, Elliott MW. Assisted ventilation: domiciliary non-invasive ventilation for recurrent acidotic exacerbations of COPD: an economic analysis. Thorax. 2003;58:867-871.

10. Budweiser S, Jorres RA, Pfeifer M. Treatment of respiratory failure in COPD. Int J COPD. 2008;3(4):605-618.

11. Series F, Marc I, Cormier Y, La Forge J. Long-term effects of protriptyline in patients with chronic obstructive pulmonary disease. Am Rev Respir Dis. 1993;147(6):1487-1490.

12. MHRA drug safety update on modafinil. MHRA Website. Mar 2011; 4(8):A1-A2.

13. [No authors listed] Managing excessive daytime sleepiness in adults. Drug Ther Bull. 2004;42:52-56.

14. Guilleminault C, Shergill RP. Sleep-disordered breathing in neuromuscular disease. Curr Treat Options Neurol. 2002;4;107-112.

15. [No authors listed] Randomized trial of modafinil as a treatment for the excessive daytime somnolence of narcolepsy: US Modafinil in Narcolepsy Multicenter Study Group. Neurology. 2000;54;1166-1175.

16. Mignot E, Nishino S, Guilleminault C, Dement WC. Modafinil binds to the dopamine uptake carrier site with low affininty. Sleep. 2004; $17: 436-437$.

17. Lin JS, Hou Y, Jouvet M. Potential brain neuronal targets for amphetamine-, methylphenidate-, and modafinil-induced wakefulness, evidenced by c-fos immunocytochemistry in the cat. Proc Natl Acad Sci U S A. 1996;93:14128-14133.
18. Engber TM, Koury EJ, Dennis SA, Miller MS, Contreras PC, Bhat RV. Differential patterns of regional c-fos induction in the rat brain by amphetamine and the wakefulness promoting drug modafinil. Neurosci Lett. 1998;241:95-98.

19. Williams SC, Rogers NL, MarshallNS, Leung S, StarmerGA, GrunsteinRR. The effect of modafinil following acute CPAP withdrawal: a preliminary study. Sleep Breath. 2008;12:359-364.

20. Warot D, Corruble E, Payan C, Weil JS, Puech AJ. Subjective effects of modafinil, a new central adrenergic stimulant in healthy volunteers; a comparison with amphetamine, caffeine and placebo. Eur Psychiatry. 1993;8:201-208

21. Kingshott RN, Vennelle M, Coleman EL, Engleman HM, Mackay TW, Douglas NJ. Randomized, double-blind, placebo-controlled crossover trial of modafinil in the treatment of residual excessive daytime sleepiness in the sleep apnea/hypopnea syndrome. Am J Respir Crit Care Med. 2001;163(4):918-923.

22. Modldofsky H, Broughton RJ, Hill JD. A randomised trial of long term, continued efficacy and safety of modafinil in narcolepsy. Sleep Med. 2000;1:109-116.

23. Broughton RJ, Fleming JAE, George CFP, et al. Randomised, doubleblind, placebo-controlled crossover trial of modafinil in the treatment of excessive daytime sleepiness in narcolepsy. Neurology. 1997;49: 444-451.

24. [No authors listed] Randomized trial of modafinil for the treatment of pathological somnolence in narcolepsy. US Modafinil in Narcolepsy Multicenter Study Group. Ann Neurol. 1998;43:88-97.

25. Wadman M. New cures sought from old drugs. Nature. 2012.490: 15-18.

26. Padhy BM, Gupta YK. Drug discovery. Technol Rev. 2011;57: 153-160.

27. DiMasi JA, Hansen RW, Grabowski HG. The price of innovation; new estimates of drug development costs. J Health Econ. 2003;22(2): $151-185$.

28. Huang R, Southall N, Wang Y, et al. The NCGC pharmaceutical collection: a comprehensive resource of clinically approved drugs enabling repurposing and chemical genomics. Sci Transl Med. 2011;3(80):80ps16.

29. Palumbo A, Facon T, Sonneveld P, et al. Thalidomide for treatment of multiple myeloma; 10 years later. Blood. 2008;111(8):3968-3977.

30. Penaloza RA, Sarkar U, Claman DM. Trends in on-label and off-label modafinil use in a nationally representative sample. JAMA. 2013;173(8): 704-706.

31. Cahill M, Balice-Gordon R. The ethical consequences of modafinil use. Penn Bioethics J. 2005;1(1):1-3.

32. Wagenaar M, Vos P, Heijdra Y, Teppema L, Folgering H. Comparison of acetazolamide and medroxyprogesterone as respiratory stimulants in hypercapnic patients with COPD. Chest. 2003;123:1450-1459.

33. Zhang J, Wang Y, Feng J, Sun X. Sleep-induced hypoxaemia in patients with chronic obstructive pulmonary disease. Brit J Hosp Med. 2013;74(9):497-502.
International Journal of COPD

\section{Publish your work in this journal}

The International Journal of COPD is an international, peer-reviewed journal of therapeutics and pharmacology focusing on concise rapid reporting of clinical studies and reviews in COPD. Special focus is given to the pathophysiological processes underlying the disease, intervention programs, patient focused education, and self management protocols.

\section{Dovepress}

This journal is indexed on PubMed Central, MedLine and CAS. The manuscript management system is completely online and includes a very quick and fair peer-review system, which is all easy to use. Visi http://www.dovepress.com/testimonials.php to read real quotes from published authors. 